\title{
Covid-19 mycotic-related rash may not be connected to steroid use
}

\section{Pardis Tabaee Damavandi}

\author{
Author Note \\ Subject to copyright law 2021@. The author is the only detainer of the copyright and intellectual property of the \\ manuscript. The manuscript cannot be reproduced nor used without citation or due acknowledgement
}




\begin{abstract}
An article earlier in the month explains that the Mucormycosis cases observed in patients with the Indian variant may be connected to excessive steroid use. Although steroid use is generally detrimental for individuals due to the wide spectrum inhibitions, the development of the rash is not necessarily connected to a suppression of immunity, but exposure to the variant may be sufficient.
\end{abstract}




\section{Covid-19 mycotic-related rash may not be connected to steroid use}

Several patients with covid-19 present into clinics, general practitioner practices, pharmacies, or hospitals, with rashes. As most viruses, coronaviruses too manifest with skin rashes. The peculiarity with the coronavirus appearance is the exhibition of a visual purple-blue rash that can be noticed at the extremities of either or both fingers and toes. The cyanotic-purple aspect to the digits also extends from the distal finger or toe towards the proximal one and is often symmetric (digits demonstrate this rash symmetrically, a similar presentation to cyanotic Raynaud's Disease), but sometimes it can only be grasped on a single digit and is usually seen post-infection. These rashes are tender to the touch and are persistent, however, other covid19 rashes can also be detected, namely vesicular-pustular rashes, palmar erythema with dried scaly skin which has been shown in patients who exhibited excessive washing and detergent use, accompanied with discomfort and no pruritus, substantially a dermatitic response to the viral agent, comparatively to other viral breakouts, and more recently, Mucormycotic types of expressions. Often, these displays are “escorted" by the presence of neighbouring swollen lymphnodes.

\section{Background and Discussion}

Patients presenting with the covid19 rashes are not always immune-compromised, and in fact in notably good health. A patient who had contracted covid19 but was asymptomatic displaying the rash on the four toes of each foot whilst travelling, had been applying butoconazole locally, without any improvements. The patient as did other patients presenting with covid19 had never been knowingly exposed to steroids.

Mucormycoses like most fungi inhabit the most humid regions, their survival is independent of the use of steroids, but dependent on the amount of water present in the environment. It makes sense that diabetic patients develop mucormycoses due to water retention 
somewhere in their body because of osmotic pressure changes deriving from the glucose in excess, making water accumulate at more "porified" areas, since leakage is higher in fenestrated capillaries where fluid exchange is maximal compared to the bigger vessels, the rashes can be evident on those anatomical surfaces from which they start and then propagate to cover a wider area. Humid soils are the most common sources of contamination for spores, as well as humid air. Nevertheless, multiple thrombotic events due to platelet activation or excessive immune responses in the peripheral areas can also be an incentive, regardless of steroid immuno-suppression, they are a consequence of the virus itself. Untreated covid19 infection may be the cause of the secondary infections. Between viruses and fungi, fungi are the more intelligent microorganisms, as they don't resort to mutating partially their viral code as viruses do in response to environmental precarious conditions, but they are able to mimic other pathogens, which is very peculiar behavior for fungi not for the virus. ${ }^{1}$

It's thus easy to also believe as an alternative theory, that all the fungi causing the rashes, are exploitative of the virulence and "omnipresence" of the coronavirus, by using the proteic-amino acidic nutrients contained in the virus, to spread easily in a symbiotic manner as the virus. Conversely, both the water leakage from the capillaries and thrombotic "background" as well as the constant presence of the virus systemically may contribute to fungal infections of any sort.

\section{Treatment}

Treatment thus could lie in a combination therapy which makes the environment of the fungus inhabitable. Clearly, an anti-fungal agent may not suffice, but a combination with anti-thrombotic, whereby thrombosis has been detected, and a peripheral vasodilator such as cilostazol, associated with anti-coronaviral therapy could be co-administered. It is true that steroid use could exacerbate fungal infections, due to the water retention induced by i.e. glucocorticoid management, however, 
it is not the cause, it may simply worsen the symptoms. This is because a lot of the patients who displayed post-infection rashes are young to middle-aged, in good health, and without any steroid use in their clinical history upon presentation. 


\section{References}

Moodley A., Mosam A., Govender N. P. et al. (2019) Emergomyces africanus: The Mimicking Fungus. Dermatopathology, 26;6(2):157-162. doi: 10.1159/000497608. PMID: 31700857; PMCID: PMC6827449. 
